Objectives: To determine whether baseline (BL) characteristics or early responses predict clinical response at 1 year in UPA-treated patients with AS. Methods: In the double-blind, randomized, placebo (PBO)-controlled SELECT-AXIS 1 study, patients received UPA $15 \mathrm{mg}$ once daily or PBO until Week 14. ${ }^{1}$ At Week 14, PBO-treated patients switched to UPA $15 \mathrm{mg}$; patients originally randomized to UPA continued UPA therapy. Data from patients in the PBO and UPA arms were combined based on overall exposure to UPA; in the switch arm, exposure was defined as current visit minus 14 weeks (time of switch). The following outcomes were assessed at 1 year: Ankylosing Spondylitis Disease Activity Score with C-reactive protein (ASDAS[CRP]) inactive disease (ID; <1.3) and low disease activity (LDA; <2.1), Assessment of SpondyloArthritis International Society (ASAS) partial remission (PR), and $\geq 40 \%$ improvement in ASAS criteria (ASAS40) response. The ability of BL characteristics, efficacy at Week 12, and back pain at Week 12 to predict 1-year outcomes was assessed using a univariable logistic regression model generating odds ratios (ORs; 95\% confidence intervals). LASSO regression was used to select the best-fitted multivariable model at Week 12 for each outcome measure.

Results: Among 187 patients who received or switched to UPA $15 \mathrm{mg}, 70$ (37.4\%), 134 (71.7\%), 73 (39.0\%), and 131 (70.1\%) achieved ASDAS(CRP) ID, ASDAS(CRP) LDA, ASAS PR, and ASAS40, respectively, following 1 year of UPA treatment. No meaningful predictors of 1-year efficacy outcomes were identified based on BL demographics (including disease duration, gender, and human leukocyte antigen B27 status) or BL disease characteristics (including ASDAS, Bath Ankylosing Spondylitis Disease Activity Index, and CRP levels). In univariable analyses, Week 12 responses based on several disease activity measures and patient-reported outcomes (PROs), including reductions (much better improvement $[\mathrm{MBI}], \geq 30 / \geq 50 / \geq 70 \%$ reduction, or improvement) in back pain score, along with lower scores for back pain at Week 12, were associated with the achievement of ASDAS(CRP) ID, ASDAS(CRP) LDA, ASAS PR, and ASAS40 at 1 year (Figure 1). In a multivariable analysis, improvement from BL to Week 12 in back pain score consistently predicted several efficacy outcomes at 1 year.

Conclusion: In upadacitinib-treated patients with AS, improvement in PROs and reduction in back pain score at 12 weeks predicted clinical outcomes at 1 year. REFERENCES:

[1] van der Heijde D, et al. Lancet 2019;394:2108-17.
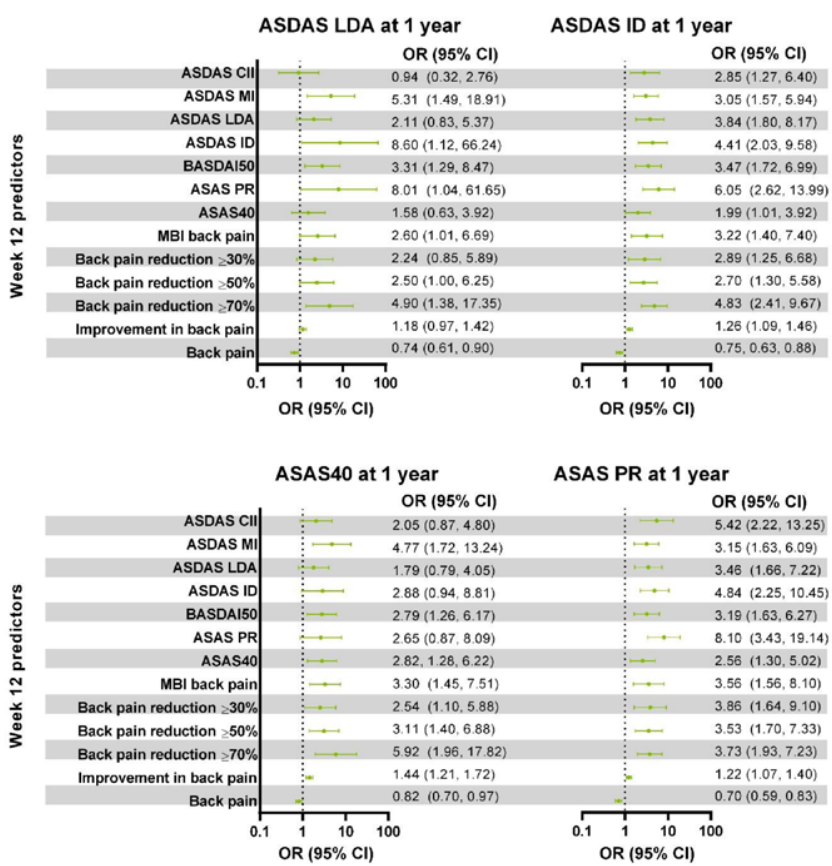

Figure 1. Association between Week 12 response or back pain at Week 12 and achievement of efficacy outcomes at 1 year (univariable analysis)

All ASDAS scores are calculated using C-reactive protein ASDAS CIl: change from $B L \geq 1.1$; ASDAS MI: change from $B L \geq 2.0$; $M B I$ back pain: $\geq 2$-point reduction in absolute score and $\geq 33 \%$ reduction from $B L$ on a $0-10$ NRS
ASAS, Assessment of SpondyloArthritis International Society; ASAS40, $\geq 40 \%$ improvement in ASAS criteria; ASDAS, Ankylosing Spondylitis Disease Activity Score; BASDAI50, $\geq 50 \%$ improvement in the Bath Ankylosing Spondylitis Disease Activity Index; $\mathrm{BL}$, baseline; $\mathrm{Cl}$, confidence interval; CII, clinically important improvement; ID, inactive disease; LDA, low disease activity; $\mathrm{MBI}$, much bette improvement; MI, major improvement; NRS, numeric rating scale; OR, odds ratio; PR, partial remission

Acknowledgements: AbbVie funded this study; contributed to its design; participated in data collection, analysis, and interpretation of the data; and participated in the writing, review, and approval of the abstract. No honoraria or payments were made for authorship. Medical writing support was provided by Laura Chalmers, PhD, of 2 the Nth (Cheshire, UK), and was funded by AbbVie.

Disclosure of Interests: Marina Magrey Consultant of: Consultant for Janssen and Novartis; member of advisory boards for Eli Lilly, Janssen, Novartis and UCB, Grant/research support from: AbbVie, Sofia Ramiro Consultant of: AbbVie, Eli Lilly, MSD, Novartis, Sanofi, and UCB, Grant/research suppor from: MSD, Marcelo Pinheiro Consultant of: AbbVie, Eli Lilly, Janssen, and Novartis, Tianming Gao Employee of: AbbVie employee and may own stock or options, Fabiana Ganz Employee of: AbbVie employee and may own stock or options, In-Ho Song Employee of: AbbVie employee and may own stock or options, Ana Biljan Employee of: AbbVie employee and may own stock or options, Nigil Haroon Consultant of: AbbVie, Amgen, Eli Lilly, Janssen, MSD, Novartis, Pfizer, and UCB, Martin Rudwaleit Consultant of: AbbVie, Bristol-Myers Squibb, Celgene, Eli Lilly, Janssen, MSD, Novartis, Pfizer, Roche and UCB

DOI: 10.1136/annrheumdis-2021-eular.1986

\section{POS0925 \\ EFFICACY OF SECUKINUMAB IN TNFI-NAÏVE PATIENTS ACROSS THE AXIAL SPONDYLOARTHRITIS SPECTRUM OVER 52 WEEKS: A POST HOC ANALYSIS OF THE MEASURE AND PREVENT CLINICAL TRIALS}

M. Magrey ${ }^{1}$, J. A. Walsh ${ }^{2}$, F. Huang ${ }^{3}$, H. Kameda ${ }^{4}$, J. Wang ${ }^{5}$, C. Herrem ${ }^{6}$, P. Pertel ${ }^{7}$, H. Marzo-Ortega ${ }^{8} .{ }^{1}$ Case Western Reserve University School of Medicine, Division of Rheumatology, Ohio, United States of America; ${ }^{2}$ Salt Lake City Veteran Affairs Medical Center; University of Utah Medical Center, Salt Lake City, Division of Rheumatology, Utah, United States of America; ${ }^{3}$ Chinese PLA General Hospital, Beijing, Department of Rheumatology and Immunology, Beijing, China; ${ }^{4}$ Toho University (Ohashi Medical Center), Department of Internal Medicine, Division of Rheumatology, Tokyo, Japan; ${ }^{5}$ Novartis Pharmaceuticals Corporation, Immunology, Hepatology and Dermatology, East Hanover, United States of America; ${ }^{6}$ Novartis Pharma AG, Rheumatology, Basel, Switzerland; ${ }^{7}$ Novartis Pharma AG, Immunology, Hepatology and Dermatology, Basel, Switzerland; ${ }^{8}$ NIHR Leeds Biomedical Research Centre, Leeds Training Hospitals NHS Trust and LIRMM, University of Leeds, NIHR Leeds Musculoskeletal Biomedical Research Unit, Leeds, United Kingdom

Background: Secukinumab (SEC) has demonstrated significant efficacy in patients (pts) with ankylosing spondylitis (AS) and non-radiographic axial spondyloarthritis (nr-axSpA). ${ }^{1-5}$ However, evidence regarding the efficacy of SEC across the axSpA disease spectrum is limited.

Objectives: To assess the efficacy of SEC on key efficacy endpoints in pts with axSpA (nr-axSpA and AS) over 52 weeks from 6 pooled phase 3 clinical trials. Methods: This post hoc analysis included pooled data from tumour necrosis factor inhibitors (TNFi)-naïve pts in the MEASURE 1-5 (AS) and PREVENT (nr-axSpA) phase 3 trials. Assessments included proportion of patients achieving ASAS40, ASAS 5/6, ASAS partial remission (ASAS-PR), ASDAS-CRP major improvement, ASDAS-CRP inactive disease (ASDAS-CRP ID), BASDAI50 and mean change from baseline in nocturnal back pain and morning stiffness with any SEC $150 \mathrm{mg}$ or SEC $300 \mathrm{mg}$ or placebo (PBO) at Week 16 , and sustainability was measured in pts treated with SEC at Week 52. Data are reported as non-responder imputation. Results: A total of 1558 TNFi-naïve pts (SEC $150 \mathrm{mg}, \mathrm{N}=932$; SEC $300 \mathrm{mg}$ $\mathrm{N}=57$ and $\mathrm{PBO}, \mathrm{N}=569$ ) with a mean age of $39.3 \pm 11.58$ years were included in the study. At Week 16, significantly higher improvements in pts treated with SEC versus PBO were observed in all efficacy endpoints (Table 1) with improvements sustained up to Week 52

Conclusion: SEC $300 \mathrm{mg}$ and $150 \mathrm{mg}$ provided significant and sustained improvement in the signs and symptoms of TNFi-naïve pts with axSpA regardless of radiographic status.

\section{REFERENCES:}

[1] Baeten D, et al. N Engl J Med 2015;373:2534-48.

[2] Pavelka K, et al. Arthritis Res Ther 2017;19:285

[3] Kivitz AJ, et al. Rheumatol Ther 2018;5:447-62.

[4] Feng H, et al. Chin Med J 2020;133:2521-31.

[5] Deodhar A, et al. Arthritis Rheumatol 2021;73:110-20. 
Table 1. Efficacy endpoints at Week 16 and Week 52 in TNFi-naïve patients with axSpA

\begin{tabular}{|c|c|c|c|c|}
\hline Endpoints & Week & $\begin{array}{l}\text { Any secuki- } \\
\text { numab } 150 \mathrm{mg} \\
(\mathrm{N}=932)\end{array}$ & $\begin{array}{c}\text { Secukinumab } \\
\begin{array}{c}\mathbf{3 0 0} \mathrm{mg} \\
(\mathrm{N}=\mathbf{5 7})\end{array}\end{array}$ & $\begin{array}{l}\text { Placebo } \\
(\mathrm{N}=569)\end{array}$ \\
\hline \multirow[t]{2}{*}{ ASAS40, n (\%) } & 16 & $399(42.8)^{\dagger}$ & $25(43.9)^{\dagger}$ & $134(23.6)$ \\
\hline & 52 & $508(54.5)$ & 33 (57.9) & - \\
\hline \multirow[t]{2}{*}{ ASAS $5 / 6, n(\%)$} & 16 & $414(44.4)^{\dagger}$ & $24(42.1)^{\dagger}$ & $125(22.0)$ \\
\hline & 52 & $508(54.5)$ & 33 (57.9) & - \\
\hline \multirow[t]{2}{*}{ ASAS partial remission, $\mathrm{n}(\%)$} & 16 & $166(17.8)^{\dagger}$ & $12(21.1)^{\dagger}$ & $35(6.2)$ \\
\hline & 52 & $240(25.8)$ & $13(22.8)$ & - \\
\hline \multirow[t]{2}{*}{ ASDAS-CRP major improvement, $\mathrm{n}(\%)$} & 16 & $266(28.5)^{\dagger}$ & $16(28.1)^{\dagger}$ & $43(7.6)$ \\
\hline & 52 & 336 (36.1) & $20(35.1)$ & - \\
\hline \multirow[t]{2}{*}{ ASDAS-CRP ID, n (\%) } & 16 & $171(18.3)^{\dagger}$ & $11(19.3)^{\dagger}$ & $31(5.4)$ \\
\hline & 52 & 247 (26.5) & $13(22.8)$ & - \\
\hline \multirow[t]{2}{*}{ BASDAI50, n (\%) } & 16 & $356(38.2)^{\dagger}$ & $22(38.6)^{\S}$ & $110(19.3)$ \\
\hline & 52 & 459 (49.2) & 27 (47.4) & - \\
\hline \multirow[t]{2}{*}{$\begin{array}{l}\text { Nocturnal back pain, mean change from } \\
\text { baseline } \pm S E\end{array}$} & 16 & $-31.82 \pm 0.87$ & $-37.99 \pm 3.56^{\dagger}$ & $\begin{array}{c}-18.12 \\
\pm 1.13\end{array}$ \\
\hline & $52^{*}$ & $-41.31 \pm 27.58$ & $-46.56 \pm 24.30$ & - \\
\hline \multirow[t]{2}{*}{$\begin{array}{l}\text { Overall level of morning stiffness, mean } \\
\text { change from baseline } \pm \text { SE }\end{array}$} & 16 & $-3.23 \pm 0.09^{\dagger}$ & $-3.62 \pm 0.35^{\dagger}$ & $\begin{array}{c}-1.95 \pm \\
0.11^{\dagger}\end{array}$ \\
\hline & $52^{*}$ & $-4.24 \pm 2.75$ & $-4.71 \pm 2.74$ & - \\
\hline
\end{tabular}

Data presented as NRI. ${ }^{\dagger} P<0.001,{ }^{\S} P<0.01$ vs placebo; ${ }^{*}$ mean change \pm SD.ASAS, Assessment of SpondyloArthritis international Society; ASDAS, Ankylosing Spondylitis Disease Activity Score; axSpA, axial spondyloarthritis; BASDAI, Bath Ankylosing Spondylitis Disease Activity Index; CRP, C-reactive protein; ID, inactive disease; NRI, non-responder imputation; SD, standard deviation; SE, standard error; TNFi, tumour necrosis factor inhibitor.

Disclosure of Interests: Marina Magrey Consultant of: Novartis, Pfizer, Abbvie, UCB, Eli Lilly, Grant/research support from: Clinical trials with Abbvie and UCB, Jessica A. Walsh Consultant of: AbbVie, Novartis, Eli Lilly and Company, UCB, Grant/research support from: AbbVie, Pfizer, Janssen, feng huang: None declared, Hideto Kameda Speakers bureau: AbbVie, Asahi-Kasei, Astellas, Bristol-Myers Squibb, Chugai, Eisai, Eli Lilly, Gilead Sciences, Janssen, Kyowa Kirin, Mitsubishi-Tanabe, Novartis, Pfizer and UCB., Consultant of: AbbVie, Asahi-Kasei, Astellas, Bristol-Myers Squibb, Chugai, Eisai, Eli Lilly, Gilead Sciences, Janssen, Kyowa Kirin, Mitsubishi-Tanabe, Novartis, Pfizer and UCB., Grant/ research support from: AbbVie, Asahi-Kasei, Astellas, Behringer, Chugai, Eisai, Mitsubishi-Tanabe, Novartis, Jianyuan Wang Employee of: Novartis, Christopher Herrem Employee of: Novartis, Patricia Pertel Employee of: Novartis, Helena Marzo-Ortega Speakers bureau: AbbVie, Celgene, Janssen, Eli Lilly and Company, Novartis, Pfizer, Takeda, and UCB., Consultant of: AbbVie, Celgene, Janssen, Eli Lilly and Company, Novartis, Pfizer, Takeda, and UCB., Grant/research support from: Janssen, Novartis

DOI: 10.1136/annrheumdis-2021-eular.2005

\section{POS0926 NORMALIZATION OF HIGH SENSITIVITY CRP VERSUS CLINICAL RESPONSE TO IXEKIZUMAB AT WEEK 16 IN PATIENTS WITH RADIOGRAPHIC \& NON-RADIOGRAPHIC AXIAL SPONDYLOARTHRITIS: RESULTS FROM THE COAST STUDIES}

H. Marzo-Ortega ${ }^{1}$, X. Juanola ${ }^{2}$, T. Okano ${ }^{3}$, Y. Schymura ${ }^{4}$, A. Bradley ${ }^{5}$, J. Gerwien ${ }^{5}$, B. Monsberger ${ }^{6}$, S. Liu Leage ${ }^{5}$, D. Aletaha ${ }^{7}$, M. Østergaard ${ }^{8}$. ${ }^{1}$ Leeds Teaching Hospitals NHS Trust \& LIRMM, University of Leeds, NIHR Leeds Biomedical Research Centre, Leeds, United Kingdom; ${ }^{2}$ University Hospital Bellvitge, Rheumatology Service, Barcelona, Spain; ${ }^{3}$ Osaka City University, Graduate School of Medicine, Osaka, Japan; ${ }^{4}$ Eli Lilly and Company, Statistics, Indianapolis, United States of America; ${ }^{5}$ Eli Lilly and Company, Medical Rheumatology, Indianapolis, United States of America; ${ }^{6} E$ Eli Lilly and Company, Dermatology, Indianapolis, United States of America; ${ }^{7}$ Medical University of Vienna, Division of Rheumatology, Wien, Austria; ${ }^{8}$ University of Copenhagen, Department of Clinical Medicine, Copenhagen, Denmark

Background: C-reactive protein (CRP), an objective marker of inflammation, can be used to monitor treatment response to biologics in patients with axial spondyloarthritis (axSpA) in addition to evaluating signs \& symptoms. CRP is not elevated in all patients with active axSpA questioning its validity as a universal biomarker of response. Ixekizumab (IXE) demonstrated efficacy in axSpA treatment irrespective of baseline (BL) CRP levels. However, response to IXE categorized on CRP change from $\mathrm{BL}$ is unknown.

Objectives: To evaluate response to IXE treatment from BL through week (wk) 16 in patients with axSpA categorized according to change in high sensitivity (hs) CRP.

Methods: COAST-V (NCT 02696785),-W (NCT02696798), \& -X (NCT02757352), were phase 3 , multicentre, randomized, controlled trials, investigating the efficacy of IXE $80 \mathrm{mg}$ every 4 \& 2 wks in patients with: r-axSpA naïve to biologic disease-modifying antirheumatic drugs (bDMARDs; COAST-V); or who were inadequate responders/intolerant to tumour necrosis factor inhibitors (TNFi;
COAST-W); or who fulfilled Assessment of SpondyloArthritis International Society (ASAS) criteria for non-radiographic (nr)axSpA (COAST-X).

This post hoc analysis focuses on approved dosing regimens. Depending on $\mathrm{BL}$ and wk 16 hsCRP values, patients were categorised as stable low (hsCRP $\leq 5 \mathrm{mg} / \mathrm{L}$ at $\mathrm{BL} \& \leq 5 \mathrm{mg} / \mathrm{L}$ at wk 16 ), normalized (hsCRP $>5 \mathrm{mg} / \mathrm{L}$ at $\mathrm{BL} \& \leq 5 \mathrm{mg} / \mathrm{L}$ at wk 16) or elevated (hsCRP $>5 \mathrm{mg} / \mathrm{L}$ at wk 16 , irrespective of $\mathrm{BL}$ hsCRP). An absolute cutoff of $5 \mathrm{mg} / \mathrm{L}$ was used as the stratification factor in all COAST studies. Data were analyzed by treatment arm. Each trial was analyzed separately.

For hsCRP subgroups, patient demographics \& other characteristics at BL, as well as trajectory over time for the endpoints ASAS40 \& Bath Ankylosing Spondylitis Disease Activity Index (BASDAI) 50 are described. Non-responder imputation was used for missing values.

Results: In all studies at BL, disease activity \& MRI SPARCC score were higher in normalized \& elevated subgroups compared to the stable low subgroup - $\mathbf{a}$. In COAST-V, ASAS40 \& BASDAI50 response was observed in IXE-treated patients regardless of hsCRP change status by wk 16 vs placebo. The highest ASAS40 \& BASDAI50 response rate was reported in patients with normalized hsCRP - b. Similar findings were observed in COAST-W \& -X

Table 1. Baseline Patient demographics \& other characteristics - ITT population, per CRP subgroup

\begin{tabular}{|c|c|c|c|c|c|c|c|c|c|}
\hline & \multicolumn{3}{|c|}{$\begin{array}{l}\text { COAST-V } \\
\text { (r-axSpA, } \\
\text { bDMARD } \\
\text { naive) }\end{array}$} & \multicolumn{3}{|c|}{$\begin{array}{c}\text { COAST-W } \\
\text { (r-axSpA, inadequate } \\
\text { responders /intolerant } \\
\text { to TNFi) }\end{array}$} & \multicolumn{3}{|c|}{$\begin{array}{c}\text { COAST-X } \\
\text { (non-radiographic } \\
\text { axSpA, bDMARD } \\
\text { naïve) }\end{array}$} \\
\hline & $\begin{array}{c}\text { Stable } \\
\text { low } \\
(n=79)\end{array}$ & $\begin{array}{l}\text { Norma- } \\
\text { lized } \\
(n=80)\end{array}$ & $\begin{array}{c}\text { Elevated } \\
(n=98)\end{array}$ & $\begin{array}{c}\text { Stable } \\
\text { low } \\
(n=58)\end{array}$ & $\begin{array}{l}\text { Norma- } \\
\text { lized } \\
(n=34)\end{array}$ & $\begin{array}{c}\text { Elevated } \\
(n=126)\end{array}$ & $\begin{array}{c}\text { Stable } \\
\text { low } \\
(n=78)\end{array}$ & $\begin{array}{l}\text { Norma- } \\
\text { lized } \\
(n=40)\end{array}$ & $\begin{array}{c}\text { Elevated } \\
(n=81)\end{array}$ \\
\hline \multicolumn{10}{|l|}{ Parameter } \\
\hline Age (years) & $\begin{array}{c}43.7 \\
(12.1)\end{array}$ & $\begin{array}{c}38.9 \\
(10.9)\end{array}$ & $\begin{array}{c}42.7 \\
(12.0)\end{array}$ & $\begin{array}{c}50.4 \\
(13.3)\end{array}$ & $\begin{array}{c}46.1 \\
(13.8)\end{array}$ & $\begin{array}{c}45.7 \\
(12.5)\end{array}$ & $\begin{array}{c}44.0 \\
(12.8)\end{array}$ & $\begin{array}{c}37.2 \\
(14.6)\end{array}$ & $\begin{array}{c}38.7 \\
(12.8)\end{array}$ \\
\hline $\begin{array}{l}\text { Male gender, } \\
\mathrm{n}(\%)\end{array}$ & $\begin{array}{c}63 \\
(79.7)\end{array}$ & $\begin{array}{l}71.0 \\
(88.8)\end{array}$ & $\begin{array}{c}78 \\
(79.6)\end{array}$ & $\begin{array}{l}39.0 \\
(67.2)\end{array}$ & $\begin{array}{c}30 \\
(88.2)\end{array}$ & $\begin{array}{c}109 \\
(86.5)\end{array}$ & $\begin{array}{c}35 \\
(44.9)\end{array}$ & $\begin{array}{c}23 \\
(57.5)\end{array}$ & $\begin{array}{c}34 \\
(42)\end{array}$ \\
\hline $\begin{array}{l}\text { Duration of } \\
\text { symptoms } \\
\text { since axSpA } \\
\text { onset (years) }\end{array}$ & $\begin{array}{c}17.5 \\
(11.2)\end{array}$ & $\begin{array}{l}14.4 \\
(9.3)\end{array}$ & $\begin{array}{l}16.1 \\
(9.9)\end{array}$ & $\begin{array}{c}21.7 \\
(12.9)\end{array}$ & $\begin{array}{l}16.8 \\
(11.6)\end{array}$ & $\begin{array}{r}18.9 \\
(10.9)\end{array}$ & $\begin{array}{l}12.1 \\
(9.9)\end{array}$ & $\begin{array}{l}10.3 \\
(9.7)\end{array}$ & $\begin{array}{l}9.5 \\
(9.0)\end{array}$ \\
\hline $\begin{array}{l}\text { HLA-B27 } \\
\text { positive, } \\
\text { n (\%) }\end{array}$ & $\begin{array}{c}69 \\
(87.3)\end{array}$ & $\begin{array}{l}75.0 \\
(93.8)\end{array}$ & $\begin{array}{c}89 \\
(90.8)\end{array}$ & $\begin{array}{c}31.0 \\
(91.2)\end{array}$ & $\begin{array}{c}101 \\
(80.2)\end{array}$ & $\begin{array}{c}101 \\
(80.2)\end{array}$ & $\begin{array}{c}48 \\
(61.5)\end{array}$ & $\begin{array}{c}31 \\
(77.5)\end{array}$ & $\begin{array}{c}67 \\
(82.7)\end{array}$ \\
\hline $\begin{array}{l}\text { BASDAI Total } \\
\text { Score }\end{array}$ & $\begin{array}{c}6.5 \\
(1.4)\end{array}$ & $\begin{array}{c}6.7 \\
(1.5)\end{array}$ & $\begin{array}{c}7.0 \\
(1.1)\end{array}$ & $\begin{array}{c}7.4 \\
(1.5)\end{array}$ & $\begin{array}{c}7.3 \\
(1.3)\end{array}$ & $\begin{array}{c}7.4 \\
(1.3)\end{array}$ & $\begin{array}{c}6.9 \\
(1.5)\end{array}$ & $\begin{array}{c}7.1 \\
(1.6)\end{array}$ & $\begin{array}{c}7.2 \\
(1.5)\end{array}$ \\
\hline $\begin{array}{l}\text { ASDAS Total } \\
\text { Score }\end{array}$ & $\begin{array}{l}3.1 \\
(0.5)\end{array}$ & $\begin{array}{l}3.9 \\
(0.6)\end{array}$ & $\begin{array}{c}4.2 \\
(0.7)\end{array}$ & $\begin{array}{l}3.5 \\
(0.6)\end{array}$ & $\begin{array}{c}4.3 \\
(0.6)\end{array}$ & $\begin{array}{l}4.4 \\
(0.8)\end{array}$ & $\begin{array}{l}3.2 \\
(0.6)\end{array}$ & $\begin{array}{c}4.2 \\
(0.8)\end{array}$ & $\begin{array}{c}4.2 \\
(0.9)\end{array}$ \\
\hline $\begin{array}{l}\text { Spinal Pain } \\
\text { due to AS }\end{array}$ & $\begin{array}{l}7.0 \\
(1.5)\end{array}$ & $\begin{array}{l}7.0 \\
(1.5)\end{array}$ & $\begin{array}{l}7.5 \\
(1.3)\end{array}$ & $\begin{array}{l}7.8 \\
(1.5)\end{array}$ & $\begin{array}{l}7.7 \\
(1.5)\end{array}$ & $\begin{array}{l}7.9 \\
(1.4)\end{array}$ & $\begin{array}{c}7.2 \\
(1.7)\end{array}$ & $\begin{array}{c}7.5 \\
(1.7)\end{array}$ & $\begin{array}{l}7.5 \\
(1.6)\end{array}$ \\
\hline \multirow[t]{2}{*}{$\begin{array}{l}\text { peripheral } \\
\text { articular } \\
\text { manifesta- } \\
\text { tions (>=1 } \\
\text { TJC or }>=1 \\
\text { SJC) }\end{array}$} & $\begin{array}{c}47 \\
(59.5)\end{array}$ & $\begin{array}{l}40 \\
(50)\end{array}$ & $\begin{array}{l}62( \\
63.3)\end{array}$ & $\begin{array}{c}43 \\
(74.1)\end{array}$ & $\begin{array}{c}24 \\
(70.6)\end{array}$ & $\begin{array}{c}86 \\
(68.3)\end{array}$ & $\begin{array}{c}56 \\
(71.8)\end{array}$ & $\begin{array}{c}33 \\
(82.5)\end{array}$ & $\begin{array}{c}66 \\
(81.5)\end{array}$ \\
\hline & & MRI & I Spine SP & ARCC S & Score & & MRI SI & IJ SPARC & CC Score \\
\hline $\begin{array}{l}\text { MRI SPARCC } \\
\text { Score }\end{array}$ & $\begin{array}{c}6.7 \\
(11.2)\end{array}$ & $\begin{array}{l}21.8 \\
(27.3)\end{array}$ & $\begin{array}{c}20.7 \\
(25.7)\end{array}$ & $\begin{array}{c}1.2 \\
(2.3)\end{array}$ & $\begin{array}{c}7.0 \\
(7.6)\end{array}$ & $\begin{array}{c}10.4 \\
(17.0)\end{array}$ & $\begin{array}{c}5.2 \\
(6.6)\end{array}$ & $\begin{array}{c}6.1 \\
(8.2)\end{array}$ & $\begin{array}{c}6.2 \\
(10.5)\end{array}$ \\
\hline
\end{tabular}

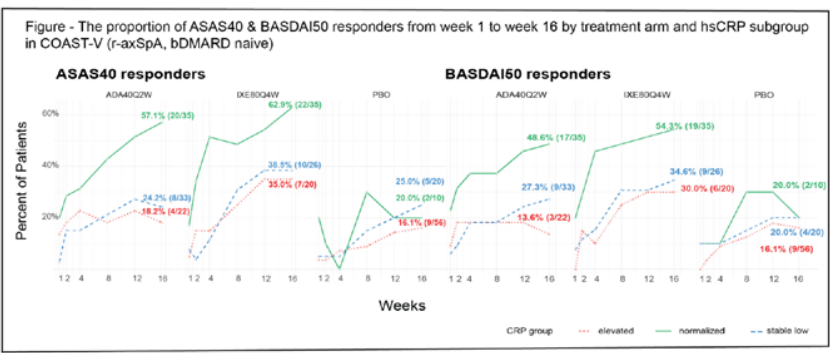

Conclusion: IXE reduced clinical disease activity in patients with axSpA irrespective of hsCRP change from BL to wk 16. Improvement in hsCRP level was associated with overall response rates.

Acknowledgements: The authors would like to acknowledge Philana Fernandes, an employee of Eli Lilly and Company, for her for writing and editorial support. Disclosure of Interests: Helena Marzo-Ortega Consultant of: Celgene, Janssen, El Lilly, Novartis, Pfizer, UCB, Grant/research support from: Janssen, Novartis, Xavie Juanola: None declared, Tadashi Okano Speakers bureau: Asahi Kasei, Astellas, Abbvie, Ayumi, Chugai, Daiichi-Sankyo, Eisai, Eli Lilly, Janssen, Mitsubishi Tanabe, 